\title{
REGULAR SEQUENCES, PROJECTIVE DIMENSION, AND CRITERIA FOR REGULARITY OF LOCAL RINGS
}

\author{
P. JOTHILINGAM AND S. MANGAYARCARASSY
}

(Communicated by Louis J. Ratliff, Jr.)

\begin{abstract}
Foxby proves the following proposition (Math. Z. 132 (1973)). Let $(R, \mathfrak{m})$ be a noetherian local ring and $M$ any finitely generated $R$-module such that the projective dimension of $M / \mathfrak{a} M$ is finite for all ideals $\mathfrak{a}$ of finite projective dimension. Then either $M$ is free or $R$ is regular local. In this article we prove that the conclusion holds if we restrict only to ideals generated by regular sequences, with the empty sequence being interpreted as the zero ideal.
\end{abstract}

Throughout, all rings are assumed to be commutative, noetherian with identity and modules finitely generated and unitary. A local ring $R$ with maximal ideal $\mathfrak{m}$ will be denoted as $(R, \mathfrak{m})$. Projective dimension will be abbreviated as p.d $\mathrm{d}_{R}$. The notation $Z(R)$ will stand for the set of zero divisors of $R$. For a local ring $R$, the complement in $\mathfrak{m}$ of $\mathfrak{m}^{2} \cup Z(R)$ will be denoted by $S$. We use [6] as a standard reference for the terminology of local algebra.

We now state our main result.

Theorem 1. Let $(R, \mathfrak{m})$ be a local ring and $M$ any finitely generated $R$-module such that $\mathrm{p} . \mathrm{d}_{R} \quad M / \mathfrak{a} M<\infty$ for all ideals a generated by regular sequences on $R$. Then either $M$ is free or $R$ is regular local.

Remark. As a convention we assume that the zero ideal is generated by the empty regular sequence. If in the statement of Theorem 1, we replace the expression "for all ideals $a$ generated by regular sequences on $R$ " by the expression "for all ideals a having finite projective dimension", then we get a theorem of Foxby proved in an appendix to his paper [3].

In proving Theorem 1, we make repeated use of the well-known "depth formula" which asserts that p.d $\mathrm{d}_{R} M+\operatorname{depth}_{R} M=\operatorname{depth} R$ for all finitely generated nonzero modules $M$ of finite projective dimension over a local ring.

We also need some lemmas.

Received by the editors November 19, 1991 and, in revised form, March 12, 1992, May 25, 1992, and July 14, 1992.

1991 Mathematics Subject Classification. Primary 13 H05.

Key words and phrases. Projective dimension, regular rings, regular sequence, discrete valuation ring.

The second author was supported by the National Board for Higher Mathematics, India. 
Lemma 1. Let $(R, \mathfrak{m})$ be a local ring and $x \in S$ any element. If $M$ is a nonzero finitely generated module over the ring $\bar{R}=R / x R$ such that $\mathrm{p} \cdot \mathrm{d}_{R} M<\infty$, then p.d $\bar{R} M<\infty$.

For the proof see [5, Corollary 27.2].

Lemma 2. Let $(R, \mathfrak{m})$ be a local ring of depth $=1$ such that $x R=y R$, for all $x$ and $y$ in $S$. Then $R$ is a discrete valuation ring.

Proof. The depth condition on $R$ implies $S \neq \varnothing$. Select any $x \in S$. If $R$ is not a DVR, clearly $\mathfrak{m} \neq x R$ and hence $\mathfrak{m}^{2}+x R \neq \mathfrak{m}$. Then by standard arguments, one can find $y \in \mathfrak{m}$ which lies outside of $\mathfrak{m}^{2}+x R$ and is not a zero divisor of $R$. But this is a contradiction; for by hypothesis $x R=y R$ and hence $y \in x R$, i.e., $y \in \mathfrak{m}^{2}+x R$. This contradiction shows that $R$ must be a DVR.

Proof of Theorem 1. Evidently we may assume $M \neq(0)$. The case of rings of zero depth poses no problem, since the "depth formula" yields p. $\mathrm{d}_{R} M=0$, which means $M$ is free.

Next, suppose depth $R=1$. Then the possibilities are either depth $M=1$ or depth $M=0$ (the depth formula is used again!). In the former case $\mathrm{p} \cdot \mathrm{d}_{R} M=0$, that is, $M$ is free. In the latter case, that is when depth $M=0$, we claim that $R$ must be a DVR. To this end, we show that given any $x \in S$, there exists a nonzero $R$-module $C$ such that (i) $x C=(0)$ and (ii) $p \cdot \mathrm{d}_{R} C / y C<\infty$ for all $y \in \mathfrak{m}$ which are not zero-divisors on $R$. Suppose, in addition, that $y$ lies in $S$. Then using Lemma 1, (ii) will show that $C / y C$ must be a free $R / y R$-module. Since $C / y C \neq(0)$, (i) will imply that $x R \subseteq y R$. Noticing that $x \notin \mathfrak{m}^{2}$, we see that $x R=y R$. This being true for all $x, y$ in $S$, we can refer to Lemma 2 and conclude that $R$ must be a DVR.

To construct the module $C$, we consider the $x$-torsion submodule $N$ of the module $M$. Clearly $N$ is the kernel of the homothety by $x^{n}$ on $M$ for some $n>0$ and hence $\mathrm{p}_{R} N<\infty$. Let $t$ be the largest nonnegative integer such that $x^{t} N \neq(0)$ and denote the module $x^{t} N$ as $C$. We show that $C$ has the properties (i) and (ii) mentioned in the previous paragraph. For, either $N=M$ or $M / N$ is $x$-torsion free. In the latter case $M / N$ will be free being of finite projective dimension and so $M \simeq N \oplus F$ for some free module $F$. This holds even if $N=M$; we have only to take $F$ to be (0). By hypothesis p. $\mathrm{d}_{R} M / y M<\infty$ for all $y \in \mathfrak{m}$ which are not zero-divisors on $R$. Then by what precedes, we see that $\operatorname{p.d}_{R} N / y N<\infty$ for all such $y$. Now considering the exact sequence,

$$
0 \rightarrow \frac{x^{t} N}{y x^{t} N} \rightarrow \frac{N}{y x^{t} N} \rightarrow \frac{N}{x^{t} N} \rightarrow 0
$$

and by what we have just proved, we see $\mathrm{p} \cdot \mathrm{d}_{R} \frac{x^{t} N}{y x^{t} N}<\infty$, i.e., $\mathrm{p} . \mathrm{d}_{R} C / y C<\infty$. This is condition (ii). That $x C=(0)$ is obvious from the definition of $C$.

This completes the proof of Theorem 1 in the case when depth $R=1$.

For the general case, we suppose now that depth $R \geq 2$. Let $x \in S$ be arbitrary and denote the ring $R / x R$ as $\bar{R}$ and the module $M / x M$ as $\bar{M}$. We show that the $\bar{R}$-module $\bar{M}$ satisfies the same hypothesis as the $R$-module $M$. For, let $\bar{x}_{1}, \bar{x}_{2}, \ldots, \bar{x}_{r}$ be any $\bar{R}$-regular sequence with preimages $x_{1}, x_{2}, \ldots, x_{r}$, respectively, in $R$. Then $x, x_{1}, x_{2}, \ldots, x_{r}$ will be a regular sequence on $R$. 
Denote the $\bar{R}$-ideal $\left(\bar{x}_{1}, \bar{x}_{2}, \ldots, \bar{x}_{r}\right)$ as $\overline{\mathfrak{a}}$ and the $R$-ideal $\left(x, x_{1}, x_{2}, \ldots, x_{r}\right)$ as $\mathfrak{a}$. (Note that $\overline{\mathfrak{a}}$ is the canonical image of $\mathfrak{a}$ under the natural homomorphism $R \rightarrow R / x R$.) Now $\bar{M} / \overline{\mathfrak{a}} \bar{M} \cong M / \mathfrak{a} M$ as $R$-modules. Since p. $\mathrm{d}_{R} M / \mathfrak{a} M$ $<\infty$ by hypothesis, we see that $\mathrm{p}^{\mathrm{d}} \mathrm{d}_{R} \bar{M} / \overline{\mathrm{a}} \bar{M}<\infty$, and by Lemma 1 , that p.d $\bar{R} \bar{M} / \overline{\mathfrak{a}} \bar{M}<\infty$. Notice that $\operatorname{depth} \bar{R}<\operatorname{depth} R$. So, using induction on depth $R$, we can conclude that either $\bar{R}$ is regular or $\bar{M}$ is $\bar{R}$-free. If $\bar{R}$ is regular for some $x \in S$, then clearly $R$ must also be regular since $x \notin \mathfrak{m}^{2}$ and there is nothing more to show. So, assume that $\bar{R}$ is nonregular for every $x \in S$. Then we have just seen that $\bar{M}$ is a free $\bar{R}$-module $\forall x \in S$. We shall prove that $M$ must be free. The case depth $M \geq 1$ is rather easy, for one can choose $x \in S$ to be a non-zero-divisor on $M$ as well. Then since $\bar{M}$ is $\bar{R}$-free, we easily see that $M$ is $R$-free. We show that the case depth $M=0$ cannot arise. Suppose, if possible, that depth $M=0$.

Claim. One can find $x \in S$ such that $M / x M$ contains a nonzero submodule $L$ of finite length. This is clear if $M$ itself has finite length; any $x \in S$ will do in this case. Suppose $M$ does not have finite length. Let $N$ be the maximal artinian submodule of $M$. Then since depth $M=0$, it follows $N \neq(0)$. Also $N \neq M$. Now, $M / N$ is a module of positive depth. Hence, we can find $x \in S$ which is not a zero-divisor on $M / N$. For this $x$, trivially the natural map $N / x N \rightarrow M / x M$ is a monomorphism. Then the submodule $N / x N$ has the required property.

Let $y \in \mathfrak{m}$ be any element such that $y$ is not a zero-divisor on $R / x R$. Such a $y$ exists since depth $R \geq 2$. Since $M / x M$ is a nonzero free $R / x R$-module, it follows that $y$ must be a non-zero-divisor on $M / x M$ and hence on the submodule $L$. This is not possible since homothety by $y$ must be nilpotent on $L$. This is a contradiction. Hence the case $\operatorname{depth} M=0$ cannot occur.

Theorem 1 is proved.

Corollary. Suppose $\mathfrak{a}$ is any nonzero ideal of the local noetherian ring $(R, \mathfrak{m})$. Assume that for every ideal $\mathfrak{b}$ generated by regular sequences on $R$, the ideal $\mathfrak{a}+\mathfrak{b}$ has finite projective dimension (in particular we are assuming $\mathbf{p} \cdot \mathrm{d}_{R} \mathfrak{a}<\infty$ ). Then $R$ is regular local.

Proof. Apply Theorem 1 to the nonfree $R$-module $R / \mathfrak{a}$.

\section{REFERENCES}

1. M. Auslander and D. Buchsbaum, Codimension and multiplicity, Ann. of Math. (2) 68 (1958).

2. N. Bourbaki, Commutative algebra, Addison-Wesley, Reading, MA, 1972.

3. H. B. Foxby, Duality homomorphisms for modules over certain Cohen-Macaulay rings, Math. Z. 132 (1973).

4. I. Kaplansky, Commutative rings, Allyn \& Bacon, Boston, MA, 1971.

5. M. Nagata, Local rings, Interscience, New York, 1962.

6. J. P. Serre, Algèbre locale-multiplicitiés, Lecture Notes in Math., vol. 11, Springer-Verlag, Berlin and New York, 1965. 